\title{
Loquat leaf extract enhances myogenic differentiation, improves muscle function and attenuates muscle loss in aged rats
}

\author{
BOKYUNG SUNG ${ }^{1}$, SEONG YEON HWANG ${ }^{1}$, MIN JO KIM $^{1}$, MINJUNG KIM ${ }^{1}$, JI WON JEONG ${ }^{1}$, \\ CHEOL MIN KIM ${ }^{3,4}$, HAE YOUNG CHUNG ${ }^{1,2}$ and NAM DEUK KIM ${ }^{1-3}$ \\ ${ }^{1}$ Department of Pharmacy, College of Pharmacy, ${ }^{2}$ Molecular Inflammation Research Center for Aging Intervention, \\ ${ }^{3}$ Research Center for Anti-Aging Technology Development, Pusan National University, Busan 609-735; \\ ${ }^{4}$ Department of Biochemistry, Pusan National University College of Medicine, Yangsan 626-770, Republic of Korea
}

Received April 11, 2015; Accepted July 3, 2015

DOI: $10.3892 / \mathrm{ijmm} .2015 .2286$

\begin{abstract}
A main characteristic of aging is the debilitating, progressive and generalized impairment of biological functions, resulting in an increased vulnerability to disease and death. Skeletal muscle comprises approximately $40 \%$ of the human body; thus, it is the most abundant tissue. At the age of 30 onwards, $0.5-1 \%$ of human muscle mass is lost each year, with a marked acceleration in the rate of decline after the age of 65 . Thus, novel strategies that effectively attenuate skeletal muscle loss and enhance muscle function are required to improve the quality of life of older subjects. The aim of the present study was to determine whether loquat (Eriobotrya japonica) leaf extract (LE) can prevent the loss of skeletal muscle function in aged rats. Young (5-month-old) and aged (18-19-month-old) rats were fed LE (50 mg/kg/day) for 35 days and the changes in muscle mass and strength were evaluated. The age-associated loss of grip strength was attenuated, and muscle mass and muscle creatine kinase (CK) activity were enhanced following the administration of LE. Histochemical analysis also revealed that LE abrogated the age-associated decrease in cross-sectional area (CSA) and decreased the amount of connective tissue in the muscle of aged rats. To investigate the mode of action of $\mathrm{LE}, \mathrm{C} 2 \mathrm{C} 12$ murine myoblasts were used to evaluate the myogenic potential of LE. The expression levels of myogenic proteins (MyoD and myogenin) and functional myosin heavy chain (MyHC) were measured by western blot analysis. LE enhanced MyoD, myogenin and MyHC expression. The changes in the expression of myogenic genes corresponded with an increase in the activity of $\mathrm{CK}$, a myogenic differentiation marker. Finally, LE activated the Akt/mammalian target of rapamycin (mTOR) signaling
\end{abstract}

Correspondence to: Dr Nam Deuk Kim, Department of Pharmacy, College of Pharmacy, Pusan National University, 63 Beon-gil 2, Busandaehag-ro, Geumjeong-gu, Busan 609-735, Republic of Korea E-mail: nadkim@pusan.ac.kr

Key words: loquat leaf extract, aging, skeletal muscle, muscle cell differentiation, Akt/mammalian target of rapamycin pathway pathway, which is involved in muscle protein synthesis during myogenesis. These findings suggest that LE attenuates sarcopenia by promoting myogenic differentiation and subsequently promoting muscle protein synthesis.

\section{Introduction}

Aging is generally associated with progressive changes in body composition and a decline in muscle strength, muscle mass (also known as sarcopenia) and aerobic capacity, leading to a reduction in mobility and an impaired quality of life in elderly subjects $(1,2)$. The reduction of force-generating capacity with increasing age is a characteristic of muscle aging. At the age of 80 , the muscle-force generating capacity is, on average, approximately $60 \%$ lower than that at the age of 20-30, and continues to decrease with age (3). Since aging is a continuous, complex process, a multitude of factors are involved in the decline in muscle force-generating capacity. The reduction in muscle mass contributes to a large proportion of the loss in muscle force-generating capacity with age. A previous study examining the anatomical cross-sectional area (ACSA) indicated that an average decrease in muslce mass of the human quadriceps femoris, elbow flexors and extensors was $<35 \%$ in aged males (68-75 years old) compared to young males (24-31 years old). Muscle fiber atrophy and muscle fiber cross-sectional area (CSA) also contributed to the age-related loss of muscle mass (4). A comparison of muscle fiber CSA between young and elderly subjects demonstrated that aging is accompanied by a $20-50 \%$ atrophy of type II muscle fibers, while type I muscle fiber size is not altered (5).

In addition to muscle atrophy, a decline in muscle regenerative capacity is associated with aging (6). It has been reported that new fiber formation could compensate for the loss of muscle mass in aged rodents; however, the regenerative capacity of muscles decreases progressively with age (6). The number and regenerative ability of progenitor cells decline during aging due to age-related changes in endocrine factors that alter their myogenic potential (7). Based on the above-mentioned impact of age-related skeletal muscle changes on the quality of life of elderly subjects, a deeper understanding of muscle aging and the development of adequate strategies to maintain muscle function are urgently required. 
Eriobotrya japonica (E. japonica), also known as loquat, is an evergreen fruit tree and is widely cultivated in China, Japan and Korea. The leaves, seeds and fruit of loquat are widely used as teas, food and folk medicines. The leaves of E. japonica (also known as Folium Eriobotryae) have been widely used in Korea, China and Japan, as a traditional medicine due to their beneficial effects on fever and chronic diseases, including headaches, lower back pain, dysmenorrhea, asthma, phlegm, chronic bronchitis and gastroenteric diseases (8). The dried leaf of the loquat is the part most commonly used in the treatment of diabetes mellitus (9). Various active compounds, such as triterpenes, flavonoids and tannins have been identified in the loquat leaf, and some of them have been reported to possess anti-metastatic, anti-hyperglycaemic and immunomodulatory properties (10-12). For example, triterpenes from the loquat leaf have been shown to exert anti-inflammatory properties (13-15) and have anti-inflammatory and anti-tumor properties (16). Recently, the anti-osteoporotic effects of the loquat leaves have been reported. In a previous study, the dietary supplementation with loquat leaf extract (LE) significantly prevented bone mineral density loss in ovariectomized mice and suppressed the RANKL-induced osteoclast differentiation of RAW 264.7 cells (17).

Among the triterpenoids, ursolic acid (UA) has been identified as a pharmacologically active constituent of the loquat leaf (18). Recently, Kunkel et al (19) reported that UA enhanced skeletal muscle insulin/insulin-like growth factor-I signaling and inhibited atrophy-associated skeletal muscle mRNA expression, thus suppressing muscle atrophy and stimulating muscle hypertrophy in mice. They also demonstrated that UA increased skeletal muscle mass, fast- and slow-twitch muscle fiber size, grip strength and exercise capacity (20). UA has also been shown to promote the differentiation of murine myoblasts (21), although the effects of LE on the muscles of aged animals and myoblast differentiation in vitro have not been evaluated. The aim of the present study was to investigate the effects of LE on the mass and function of muscle in aged rats. We also examined the hypothesis that LE promotes myogenic differentiation by modulating myogenesis-related gene products.

\section{Materials and methods}

Materials. All chemical reagents were obtained from Sigma-Aldrich (St. Louis, MO, USA) onless otherwise stated. 3-(4,5-Dimethylthiazol-2-yl)-2,5-diphenyltetrazolium bromide (MTT) was obtained from AMRESCO (Solon, $\mathrm{OH}, \mathrm{USA}$ ). Antibodies against myosin heavy chain (MyHC; sc-20641), MyoD (sc-760), myogenin (sc-576), phosphorylated (p-)Akt (Ser473; sc-7985-R), Akt1/2/3 (sc-8312), anti-rabbit IgG-horseradish peroxidase (HRP)-conjugated antibody (sc-2054), and anti-mouse IgG-HRP-conjugated antibody (sc-2031) were obtained from Santa Cruz Biotechnology, Inc., (Dallas, TX, USA). Antibodies against eukaryotic translation initiation factor 4E binding protein 1 (4E-BP1; no. 9452), p-4E-BP1 (Thr37/46; no. 2855), mammalian target of rapamycin (mTOR; no. 2983), p-mTOR (Ser2488; no. 5536), and p-p70 S6 kinase (Thr389; no. 9234) were purchased from Cell Signaling Technology, Inc., (Danvers, MA, USA). $\beta$-actin antibody was purchased from Sigma-Aldrich, and polyclonal antibody against p70 S6 kinase (bs-6370R) was obtained from Bioss, Inc. (Woburn, MA, USA). Dulbecco's modified Eagle's medium (DMEM) was purchased from WelGENE, Inc. (Daegu, Korea), and horse serum (HS) was from Invitrogen Life Technologies (Grand Island, NY, USA). Fetal bovine serum (FBS) and penicillin/streptomycin were purchased from GE Healthcare Life Sciences (Logan, UT, USA). A creatine kinase (CK) enzymatic assay kit (MaxDiscovery ${ }^{\circledR}$ Creatine Kinase Enzymatic Assay kit) was purchased from Bioo Scientific Corp. (Austin, TX, USA). Polyvinylidene difluoride (PVDF) membranes were obtained from Merck Millipore (Billerica, MA, USA).

Preparation of LE. An ethanol (EtOH) extract of loquat leaf (hereafter termed LE) was prepared as previously described in the study by Jung et al (22) with some modifications, and was provided by the Marine Bio-industry Development Center (Busan, Korea). The yield of LE based on the dried weight of the loquat leaf was $4.68 \%$. The final concentration of UA in the LE was $104.14 \mathrm{mg} / \mathrm{g}$. LE was dissolved in $\mathrm{EtOH}$ as a $5 \mathrm{mg} / \mathrm{ml}$ stock solution. The stock solution was stored at $-20^{\circ} \mathrm{C}$ and diluted with medium to the desired concentration prior to use.

Animal experiments. Male Sprague-Dawley rats (aged 5 and 18-19 months) were obtained from Samtako (Osan, Korea). The rats were maintained under controlled environmental conditions $\left(23 \pm 1^{\circ} \mathrm{C}, 50 \pm 10 \%\right.$ relative humidity, $12 \mathrm{~h} / 12 \mathrm{~h}$ light/dark cycle) with ad libitum access to water and a basal diet (FORMULA M07; FEEDLAB, Guri, Korea). After an acclimation period (1 week), the rats were randomly divided into 4 study groups (4-6 animals per group) as follows: i) young rats not administered LE (Y-Con group); ii) young rats administered LE (Y-LE group); iii) aged rats not administered LE (O-Con group); and iv) aged rats administered LE (O-LE group).

Animals in the LE-treated groups were administered LE mixed in their food (based on food intake, daily dose of $\mathrm{LE}=50 \mathrm{mg} / \mathrm{kg}$ of body weight). During the experiments, forelimb grip strength was determined using a grip strength meter equipped with a T-shaped pull bar (Columbus Instruments, Columbus, OH, USA). After the scheduled experiment, the rats were sacrificed by decapitation and soleus and gastrocnemius muscles were quickly removed. Muscle weights were measured immediately, and muscles were divided for biological analysis and histological examination. The animal protocol used in the present study was reviewed and approved by the Pusan National University Institutional Animal Care and Use Committee (PNU-IACUC; Approval no. PNU 2013-0461) with respect to ethical issues and scientific care.

Histological analysis. Medial portions of soleus muscles were fixed in $10 \%$ formalin solution for $24 \mathrm{~h}$, routinely embedded in paraffin blocks, transversely sectioned (3- $\mu$ m-thick sections) and stained with hematoxylin and eosin (H\&E).

Analysis of CSA. To analyze the muscle fiber CSA, images of the soleus muscle were captured using a microscope (Axiovert 100; Zeiss, Göettingen, Germany). The CSA of each muscle fiber in each field was measured using ImageJ software (version $1.49 \mathrm{~m}$, National Institutes of Health, Bethesda, MD, USA). The CSA was examined in 3 rats from each group.

CK activity assay. The cytosolic fraction of the muscle homogenate was used for the CK activity assay. Briefly, the tissues 
were homogenized in homogenate buffer containing $50 \mathrm{mM}$ HEPES (pH 7.4), $10 \mathrm{mM} \mathrm{KCl,} 2 \mathrm{mM} \mathrm{MgCl}{ }_{2}, 1 \mathrm{mM}$ DTT, $0.1 \mathrm{mM}$ EDTA, $0.1 \mathrm{mM}$ phenylmethylsulfonyl fluoride (PMSF), $20 \mathrm{mM} \beta$-glycerophosphate, $20 \mathrm{mM}$ sodium fluoride, $2 \mathrm{mM}$ sodium orthovanadate, $1 \mathrm{mM}$ pepstatin, $2 \mathrm{mM}$ leupeptin and $5 \mathrm{mM}$ aprotinin, and the homogenates obtained were placed on ice for $15 \mathrm{~min}$. Nonidet P-40 (NP-40; 10\%, $125 \mu \mathrm{l} / 1 \mathrm{ml}$ homogenate) solution was then added, mixed for $15 \mathrm{sec}$, and the mixture was then centrifuged at $14,000 \mathrm{x} g$ for $2 \mathrm{~min}$. The supernatants obtained were used as cytosolic fractions.

For the $\mathrm{CK}$ assay using the $\mathrm{C} 2 \mathrm{C} 12$ cells, the cells were collected, washed with Dulbecco's phosphate-buffered saline (DPBS) and then lysed with lysis buffer [40 mM Tris (pH. 8.0), $120 \mathrm{mM} \mathrm{NaCl}, 0.5 \%$ NP-40, $100 \mu \mathrm{g} / \mathrm{ml}$ PMSF and complete protease inhibitor] and stored at $-70^{\circ} \mathrm{C}$ until use. $\mathrm{CK}$ activity was determined using a CK enzymatic assay kit (Bioo Scientific Corp.), according to the manufacturer's instructions. Briefly, $250 \mu \mathrm{l}$ of CK reagent were added to $5 \mu \mathrm{l}$ of lysate or homogenate in a microplate. CK activity was immediately measured 2 times at 5 -min time intervals, at $340 \mathrm{~nm}$, using a multi-well reader (GENios; Tecan Austria GmbH, Grödig, Austria). The assay was performed in duplicate. The average 5 -min increase in absorbance was multiplied by 2,186 (conversion factor) to obtain the CK activity (IU/l).

Cell culture. $\mathrm{C} 2 \mathrm{C} 12$ murine myoblasts were purchased from the American Type Culture Collection (ATCC; Manassas, VA, USA). For all experiments, the $\mathrm{C} 2 \mathrm{C} 12$ cells were cultured from 4 to 9 passages to $70-80 \%$ confluence in growth medium (GM) containing DMEM supplemented with $10 \%$ FBS, $100 \mathrm{U} / \mathrm{ml}$ penicillin and $100 \mu \mathrm{g} / \mathrm{ml}$ streptomycin. The cells were maintained in humidified $95 \%$ air and $5 \% \mathrm{CO}_{2}$ at $37^{\circ} \mathrm{C}$.

For differentiation, the cells were plated at an approximate initial density of $1 \times 10^{5}$ cells/well in 6-well culture plates and grown in GM. When the cells reached $80-90 \%$ confluence, the GM was removed and the cells were washed with DPBS and fed differentiation medium (DM) containing DMEM supplemented with $2 \%$ HS to induce differentiation. To examine the effects of LE on myogenic differentiation, LE was added to the DM. The medium was changed every other day until day 6 , and the LE was replaced with each medium change.

Cell viability assay. Cell viability was evaluated by measuring the mitochondrial-dependent conversion of the yellow tetrazolium salt, MTT, to purple formazan crystals by metabolically active cells. Briefly, the $\mathrm{C} 2 \mathrm{C} 12$ cells were seeded and induced to differentiate as described above. At the end of the differentiation period, the cells were incubated with $0.5 \mathrm{mg} / \mathrm{ml}$ MTT at $37^{\circ} \mathrm{C}$ for $2 \mathrm{~h}$. Subsequently, the MTT was removed and the formazan crystals were dissolved in dimethyl sulfoxide. The absorbance was measured at $540 \mathrm{~nm}$ using a multi-well reader (Thermo Fisher Scientific, Vantaa, Finland).

Western blot analysis. Following treatment, the cells were harvested and washed with cold DPBS. The cells were lysed in lysis buffer. Following centrifugation, the supernatant was collected and the protein concentration was determined using protein assay reagents (Bio-Rad, Hercules, CA, USA). Equal amounts of protein were boiled for $5 \mathrm{~min}$ in $2 \mathrm{X}$ Laemmli sample buffer (Bio-Rad). The protein samples were then separated by sodium dodecyl sulfate-polyacrylamide gel electrophoresis on 6-15\% acrylamide gels and transferred onto PVDF membranes. The membranes were blocked with $5 \%$ non-fat dry milk in Tris-buffered saline with Tween-20 buffer (TBST; $20 \mathrm{mM}$ Tris, $100 \mathrm{mM} \mathrm{NaCl}$, pH 7.5 and $0.1 \%$ Tween-20) for $1 \mathrm{~h}$, incubated with various primary antibodies at $4{ }^{\circ} \mathrm{C}$ overnight, washed 3 times with TBST buffer and then incubated with HRP-conjugated secondary antibodies (Santa Cruz Biotechnology, Inc.) at room temperature for $1 \mathrm{~h}$. Antigen-antibody complexes were detected using an enhanced chemiluminescence (ECL) detection system (GE Healthcare Life Sciences, Piscataway, NJ, USA). Densitometric analysis (for optical density) was performed using Fluorchem SP AlphaEase ${ }^{\circledR}$ FC (version 6.0.0) software (Alpha Innotech, San Leandro, CA, USA), normalized to actin or other control proteins, and expressed as a fold change compared with the untreated controls.

Statistical analysis. Data are expressed as the means \pm SEM, and analyzed using GraphPad Prism software (version 5.02, GraphPad Software, Inc., La Jolla, CA, USA). Treatments were compared by one-way ANOVA followed by Tukey's post hoc test for pairwise comparisons. P-values $<0.05$ were considered to indicate statistically significant differences.

\section{Results}

Effects of LE supplementation on body weight in aged rats. Changes in body weight were measured every 5 days, and food and water intake were measured every 3 days over the entire experimental period. No adverse effects were observed in behavior, cleanliness and in the appearance of hair and eyes. No significant differences in food and water intake were observed between the beginning and the end of the treatment period for each experimental group (data not shown). As shown in Fig. 1A, the young-aged groups, either the control or the group fed LE, continuously gained body weight during the experimenatl period, whereas the old-aged groups showed insignificant changes in body weight.

$L E$ supplementation increases muscle strength in aged rats. We then examined the effects of LE supplementation on age-associated muscle function in rats. To monitor and quantify muscle function, we conducted a forelimb grip strength test using a grip strength meter. Grip strength was measured in each rat once every 5 days. As expected, the old-aged groups showed a significantly lower grip strength than the young-aged groups at the start of the experimental period (day 0, Fig. 1B). Within the age-matched experimental groups, no significant differences in muscle strength were observed on day 0 of the experimental period (Fig. 1B). After 35 days of LE supplementation, the mean grip strength of the old-aged control group (O-Con) decreased by $33 \%$ compared with that of the young-aged control group (Y-Con, Fig. 1C). Following LE supplementation, only an $18 \%$ decrease in grip strength was observed in the old-aged group supplemented with LE (O-LE group) compared with the Y-Con group. LE supplementation resulted in a significantly increased grip strength in the O-LE group compared with the O-Con group. The grip strength of the rats in the Y-LE group tended to slightly increase, although this change was not significant compared to the rats in the Y-Con group (Fig. 1C). 

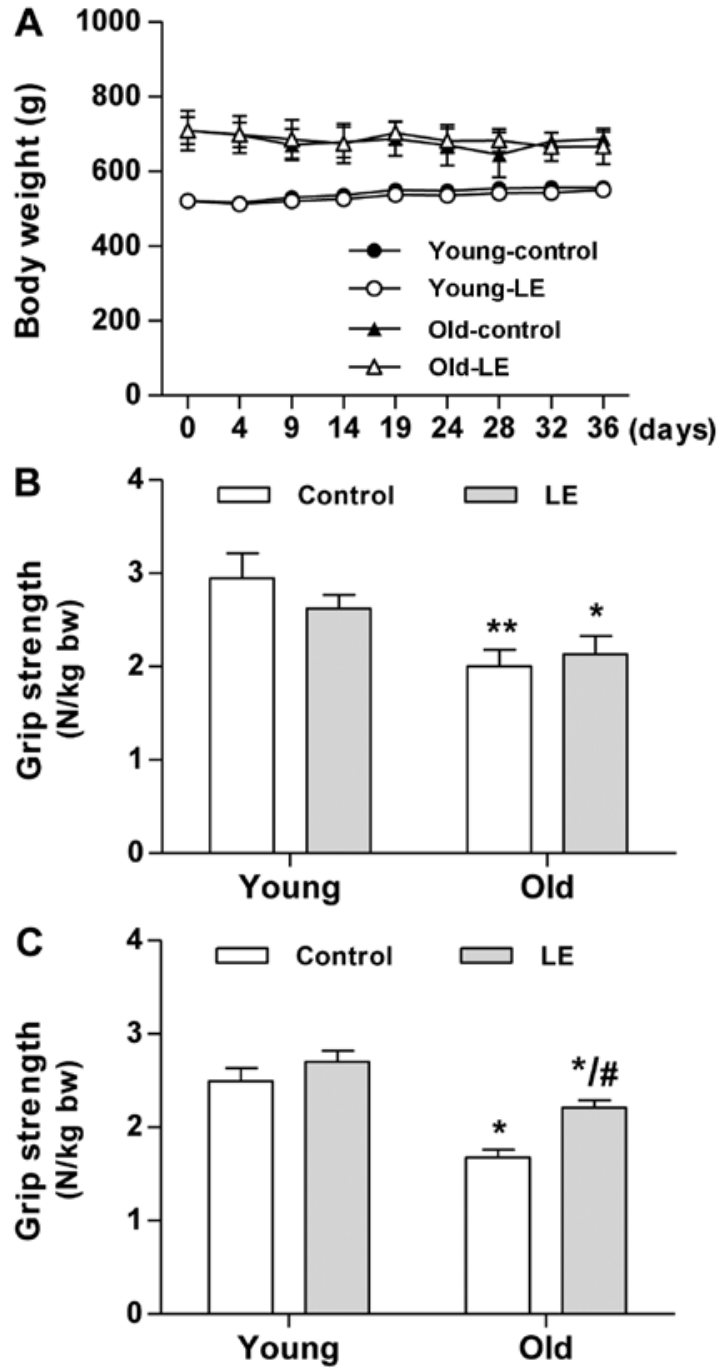

Figure 1. LE supplementation increases forelimb grip strength in rats (A) Body weight changes in each group during the experimental period. (B and C) Forelimb grip strengths in the study groups on (B) day 0 and (C) before sacrifice on day 35. Bars indicate the mean \pm SEM. ${ }^{*} \mathrm{P}<0.05$, ${ }^{* *} \mathrm{P}<0.01$ vs. young-aged controls, ${ }^{\text {} P} \mathrm{P}<0.05$ vs. old-aged controls. $\mathrm{LE}$, ethanol extract of loquat leaf; bw, body weight.

These results suggest that LE supplementation has beneficial effects on muscle strength.

LE supplementation increases muscle mass and muscle CK activity in aged rats. Grip strength decreased in the aged rats in comparison with the young ones, and we then wished to determine whether this decline in grip strength is associated with muscle mass. To determine muscle mass, the weights of the soleus and gastrocnemius muscles were measured immediately after biopsy. Aging resulted in a significant decrease in muscle mass in both the gastrocnemius (Fig. 2A) and soleus muscle (Fig. 2B). Moreover, gastrocnemius muscle mass in the O-LE group tended to increase; however, this trend was not significant compared with the O-Con group (Fig. 2A).

As regards the soleus muscle, the supplementation of LE significantly increased muscle mass in the rats in the O-LE group compared with their age-matched counterparts (Fig. 2B). However, LE intake did not significantly affect the mass of either muscle in the young-aged group (Fig. 2A
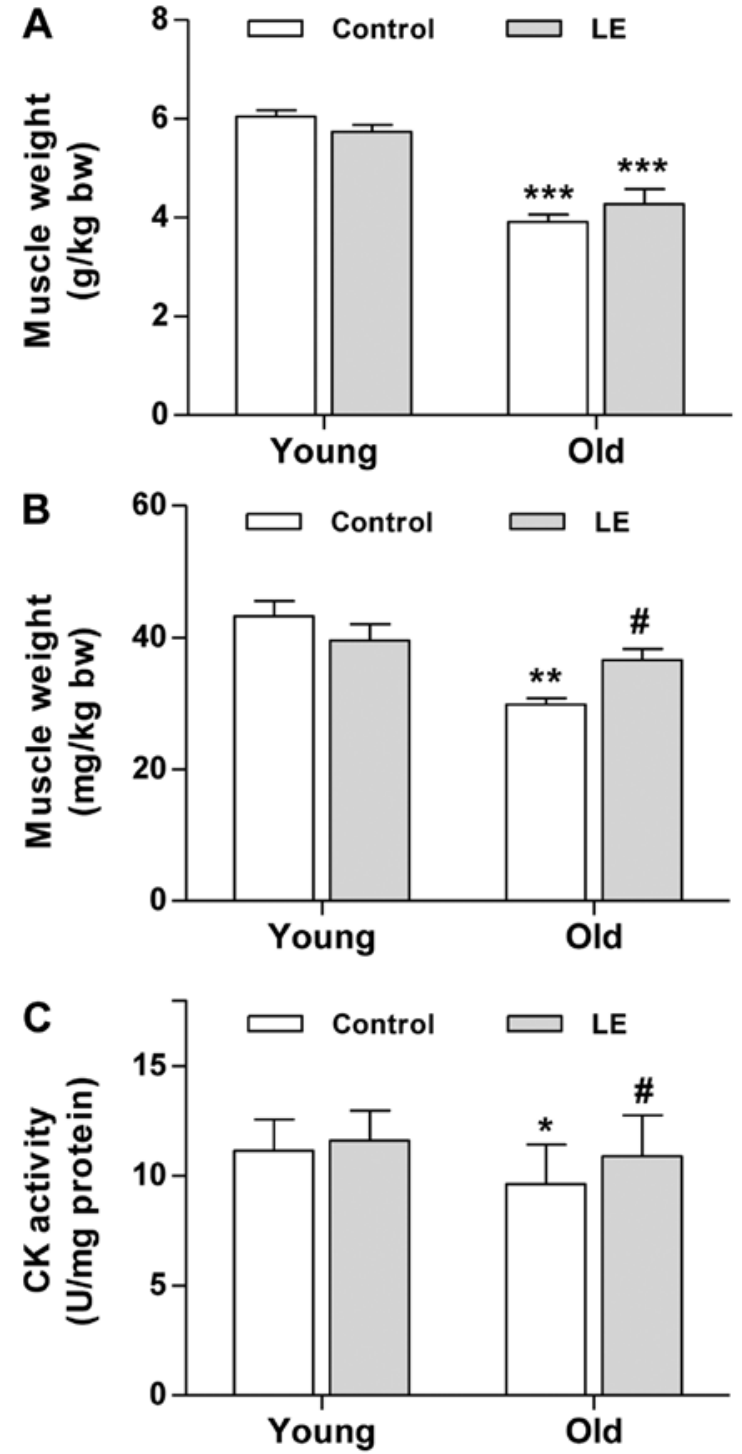

Figure 2. LE increases soleus muscle weight and creatine kinase (CK) activity. (A) Gastrocnemius and (B) soleus muscles from each rat were collected immediately after sacrifice. Muscle weights for each rat were normalized to their body weights (bw). (C) Gastrocnemius muscles were collected, homogenized and processed for $\mathrm{CK}$ assay. Bars indicate the mean $\pm \mathrm{SEM}$. ${ }^{*} \mathrm{P}<0.05,{ }^{*} \mathrm{P}<0.01$, ${ }^{* * * *} \mathrm{P}<0.001$ vs. young-aged control, ${ }^{*} \mathrm{P}<0.05$ vs. old-aged control. $\mathrm{LE}$, ethanol extract of loquat leaf.

and B). As the decrease in muscle-specific CK activity may be a major contributor to the loss of muscle function associated with aging (23), we assessed the effects of LE supplementation on $\mathrm{CK}$ activity in muscles from young and aged rats. As shown in Fig. 2C, CK activity in the young rats tended to increase slightly, although this change was not statistically significant. By contrast, aging resulted in a significant decrease in muscle CK activity (Fig. 2C). Moreover, LE intake significantly increased CK activity in the O-LE group compared with the O-Con group. Taken together, these findings suggest that LE supplementation improves muscle function in aged rats.

LE supplementation affects age-related muscle damage and muscle fiber CSA. In order to investigate the effects of LE on age-associated muscle weakness, we performed histological analysis on the medial sections of soleus muscles using H\&E 

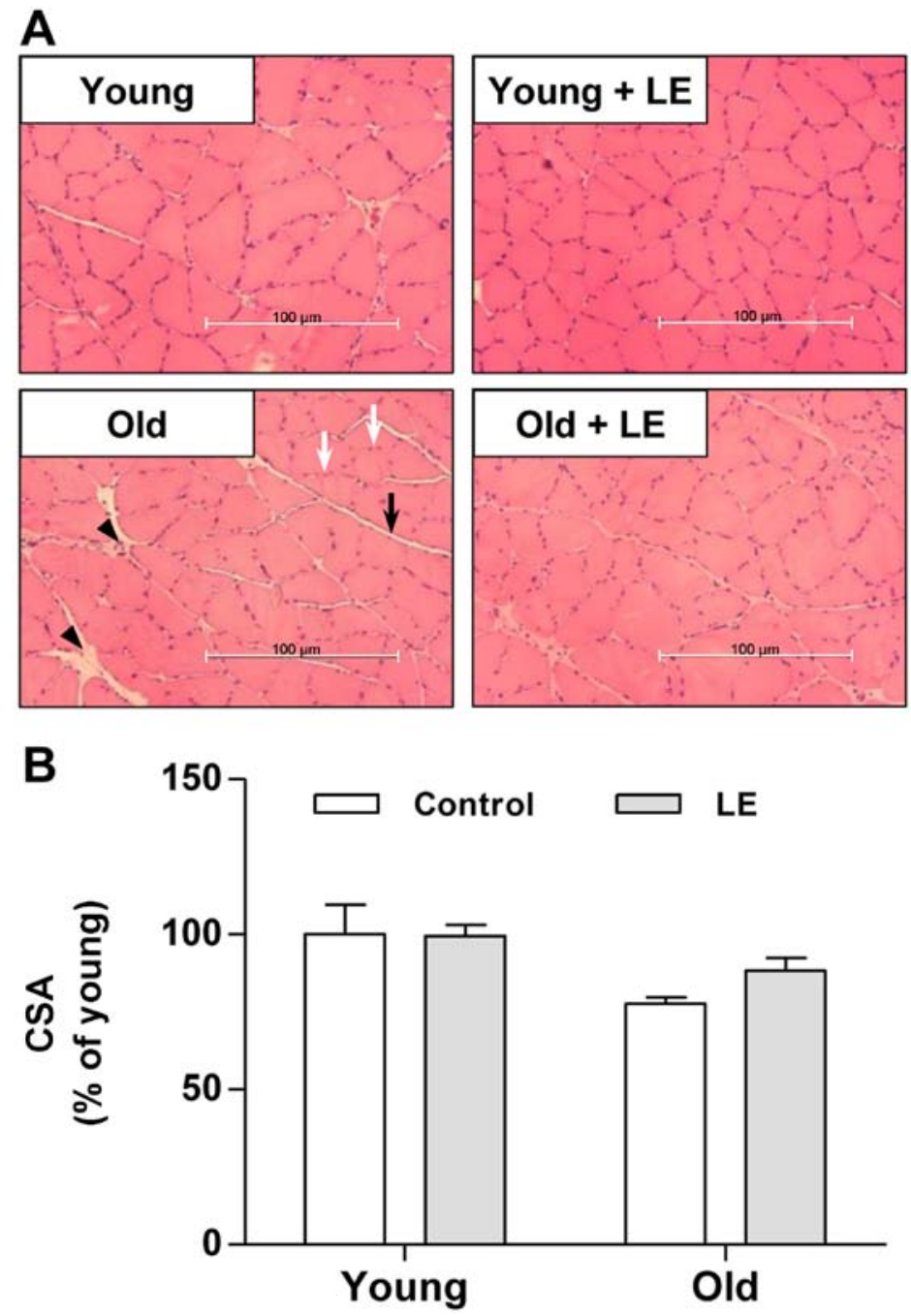

Figure 3. LE supplementation attenuates age-related damage to the soleus muscle. (A) Representative H\&E staining images of soleus muscle from each group. Original magnification, $\mathrm{x} 40$. Scale bar, $100 \mu \mathrm{m}$. Black arrow indicates the perimysium; white arrows indicate the endomysium; arrowheads indicate connective tissue. (B) Quantification of the cross-sectional area (CSA) of rat soleus muscle myofibers. Results are expressed as a relative percentage compared with muscle from young-aged control group. Values shown are the result of experiments on 3 animals per group. LE, ethanol extract of loquat leaf extract.

staining. As shown in Fig. 3A, muscle fibers in the Y-Con group were in intimate contact in muscle bundles. LE supplementation to young rats also produced similar histological results as those observed in the rats in the Y-Con group. In the aged rats, the amount of connective tissue increased compared with that in young rats (Fig. 3A, arrowhead). Thus, connective tissue surrounding the muscle fibers (endomysium) and fiber bundles (perimysium) was observed in the soleus muscle of the O-Con group compared with the young groups; however, these histological changes were attenuated by LE supplementation (Fig. 3A). We also examined the effects of aging and LE intake on muscle CSA. The CSA in the Y-LE group did not differ from that in the Y-Con group (Fig. 3B). Moreover, the CSA in the aged rats (O-Con group) was lower than that in the Y-Con group, although the difference was not statistically significant (Fig. 3B). In the aged rats, LE supplementation increased the CSA slightly, although not significantly (Fig. 3B).

LE promotes the myogenic differentiation of $\mathrm{C} 2 \mathrm{C} 12$ cells. To investigate the effects of LE on muscle regeneration, we examined whether LE affects the myogenic differentiation of $\mathrm{C} 2 \mathrm{C} 12$ myoblasts. Since it normally takes up to 6 days for $\mathrm{C} 2 \mathrm{C} 12$ myoblasts to fully differentiate into myotubes, we first assessed the effects of LE on $\mathrm{C} 2 \mathrm{C} 12$ cell viability by MTT assay under identical conditions of differentiation. As shown in Fig. 4A, LE $(0.25-2.5 \mu \mathrm{g} / \mathrm{ml})$ did not induce the significant cell death of myoblasts.

We then assessed whether LE affects the differentiation of myoblasts into myotubes. For this purpose, we used DM containing $2 \% \mathrm{HS}$. The undifferentiated $\mathrm{C} 2 \mathrm{C} 12$ cells (Fig. 4B, panel GM) were flat, fusiform or star-shaped. The myotubes began to appear 3-4 days following the induction of differentiation (data not shown). After 6 days of incubation in DM, the $\mathrm{C} 2 \mathrm{C} 12$ cells became differentiated (Fig. 4B, panel LE 0 ). The myotubes exhibited thick and fusiform structures, and they were elongated in 3-4 directions. We found that treatment with LE promoted the differentiation of the $\mathrm{C} 2 \mathrm{C} 12$ myoblasts (Fig. 4B). When the LE-treated cells (panels LE 0.1-2.5) were compared with the DM-treated cells (panel LE 0) the myotubes from the LE-treated cells were more stretched and longer in shape with syncytia and nuclei and were more abundant in number than those in the DM-treated cells. Furthermore, thick and Y-shaped 


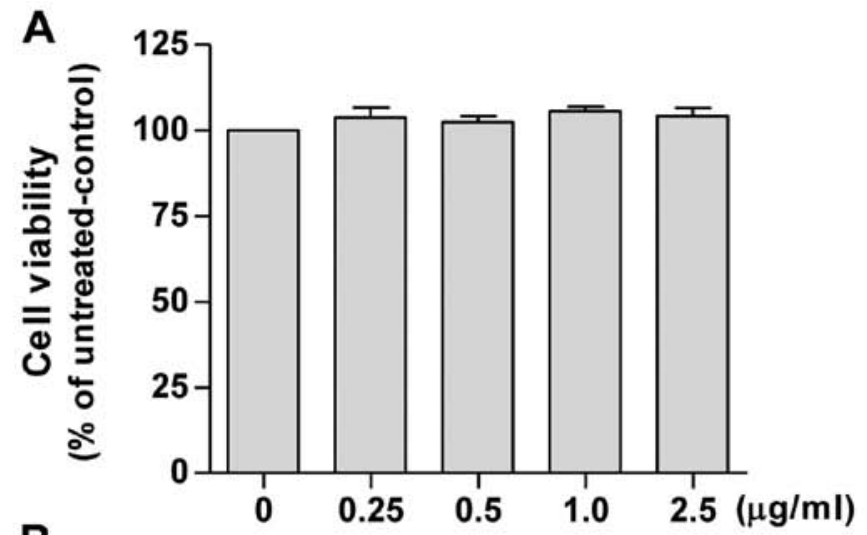

B
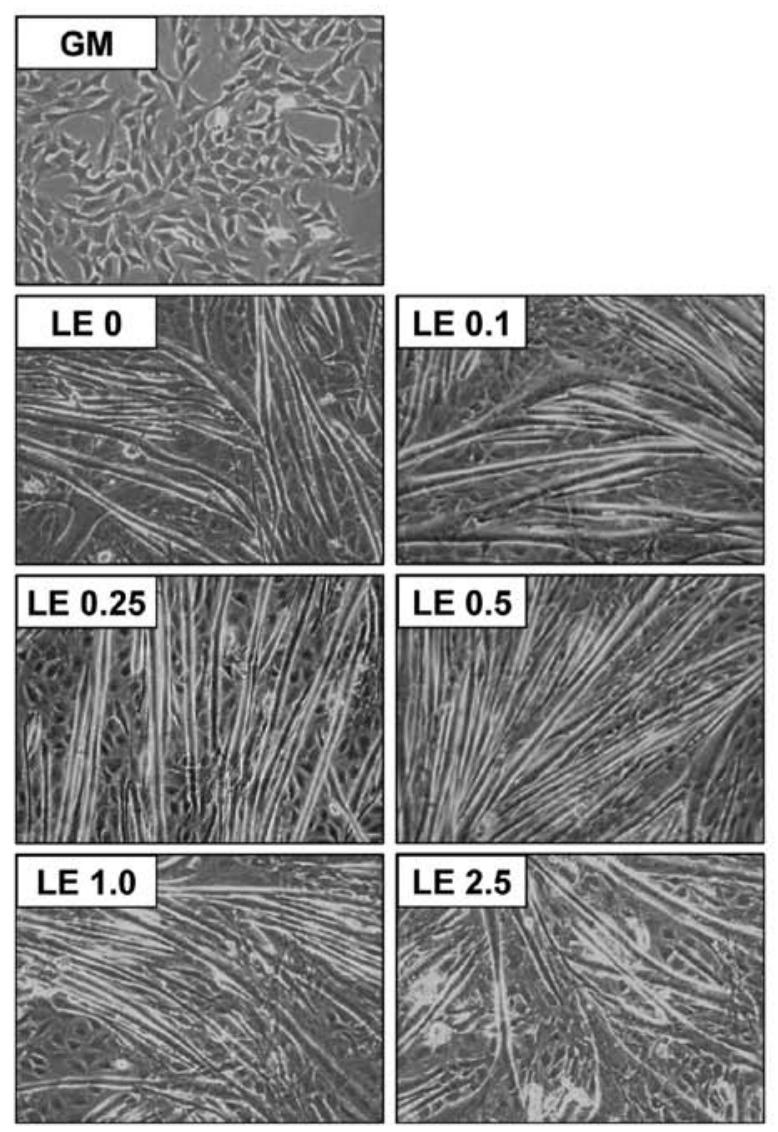

Figure 4. LE promotes the differentiation of $\mathrm{C} 2 \mathrm{C} 12$ myoblasts. (A) Changes in cell viability during the 6-day differentiation in the presence and absence of LE were measured by MTT assay. Results are expressed as the means \pm SEM of 3 independent experiments. (B) Representative images of C2C12 cells. Cells were cultured with differentiation medium (DM) in the presence and absence of LE for 6 days. LE, ethanol extract of loquat leaf; GM, growth medium.

(or spindly ring-shaped) myotubes were also occasionally observed in the $2.5 \mu \mathrm{g} / \mathrm{ml}$ LE-treated cells.

LE enhances the expression of the myogenic differentiation marker, $M y H C$, and increases $C K$ activity. To further confirm the effects of LE on myogenic differentiation, we measured the expression of the differentiation marker, $\mathrm{MyHC}$, which is the major structural protein in myotubes (24). As shown in Fig. 5A, the LE-treated C2C12 cells exhibited an increased expression of $\mathrm{MyHC}$ in a concentration-dependent manner compared with the untreated control cells.
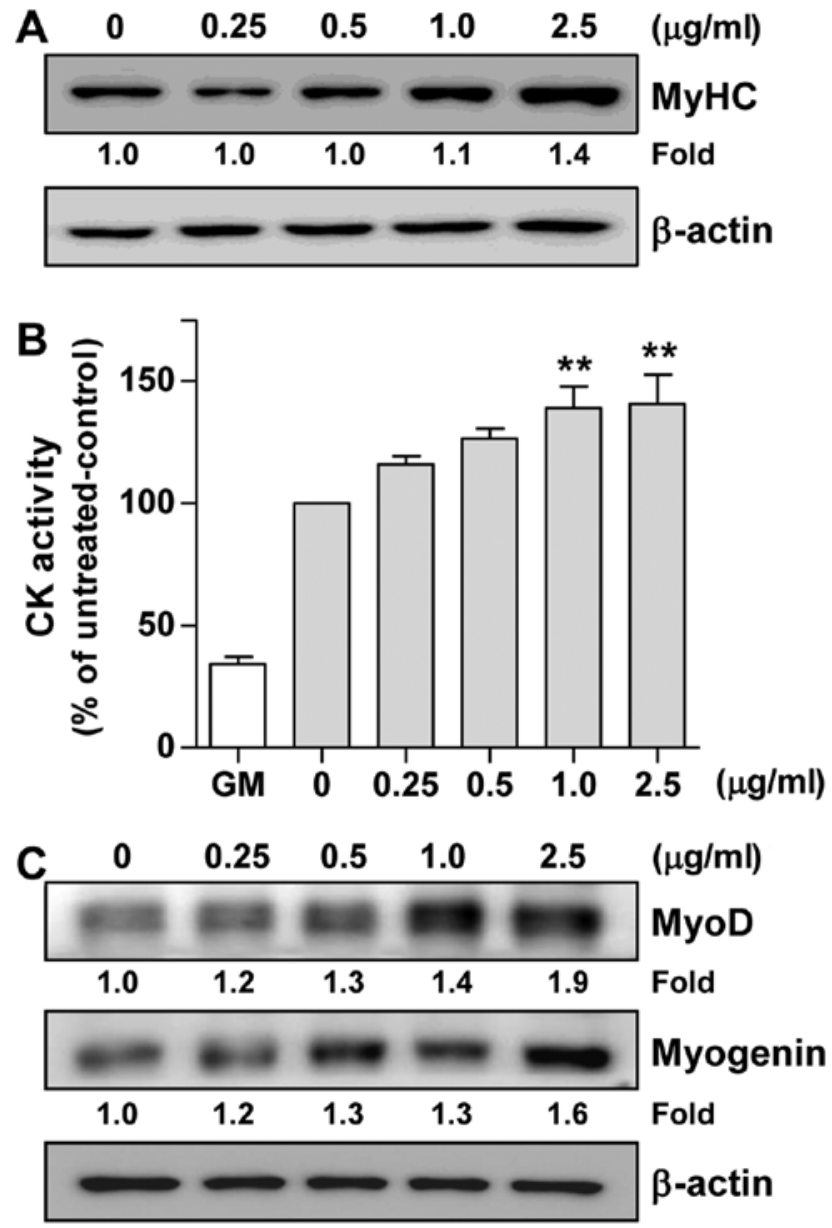

Figure 5. LE increases the expression of muscle differentiation-related genes and creatine kinase (CK) activity. (A) Western blot analysis of myosin heavy chain (MyHC) expression in $\mathrm{C} 2 \mathrm{C} 12$ cells. Cells were cultured with differentiation medium (DM) in the presence of LE for 6 days. Relative density of MyHC bands normalized to $\beta$-actin is indicated above each lane. Results are representative of 3 independent experiments. (B) Analysis of CK activity from $\mathrm{C} 2 \mathrm{C} 12$ cells induced to differentiate for 6 days in the presence and absence of LE. Results are expressed as the means \pm SEM from 3 independent experiments performed in duplicate. ${ }^{* *} \mathrm{P}<0.01$ vs. untreated control. (C) Western blot analysis of MyoD and myogenin expression in $\mathrm{C} 2 \mathrm{C} 12$ cells. Cells were cultured with $\mathrm{DM}$ in the presence of LE for $24 \mathrm{~h}$. Relative density of bands normalized to $\beta$-actin is indicated above each lane. Results are representative of 3 independent experiments. LE, ethanol extract of loquat leaf; GM, growth medium.

We then assessed CK activity, which is generally accepted as an indicator of the differentiation state of muscle cells (25). Since CK activity gradually increased until reaching peak levels on day 6 post-differentiation (data not shown), we measured $\mathrm{CK}$ activity on day 6 of differentiation. As shown in Fig. 5B, CK activity was induced 2.9-fold by DM alone ( $0 \mathrm{mg} /$ $\mathrm{ml}$ bar) in comparison with GM. In addition, compared to treatment with DM alone (0 $\mathrm{mg} / \mathrm{ml}$ bar), treatment with LE significantly enhanced $\mathrm{CK}$ activity in a concentration-dependent manner (Fig. 5B). Therefore, these results suggest that LE enhances myoblast differentiation into myotubes by increasing $\mathrm{CK}$ activity and upregulating MyHC expression.

$L E$ increases the expression of $M y o D$ and myogenin in $C 2 C 12$ cells. To elucidate the mechanisms of myogenic differentiation induced by LE, we examined the effects of LE on the levels of myogenic regulatory factors (MRFs). Western 


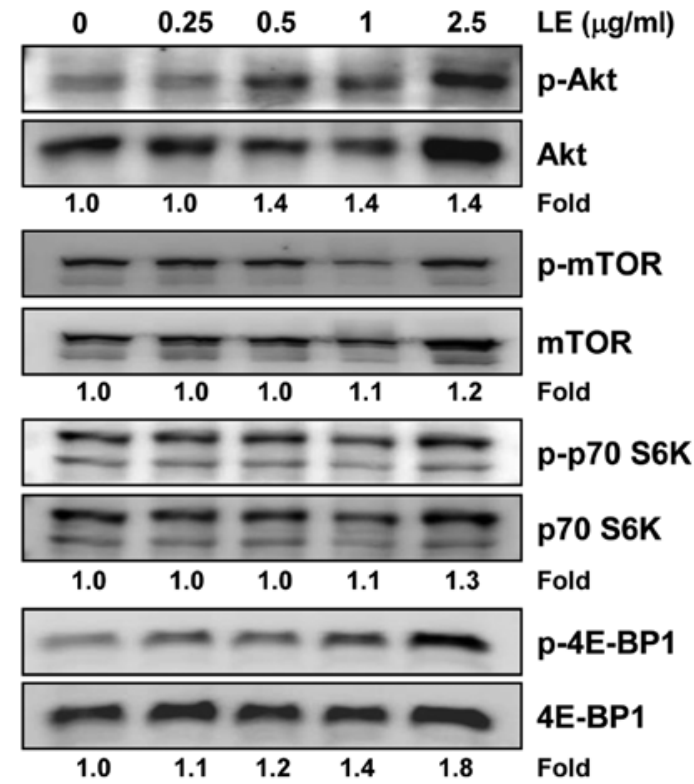

Figure 6. LE modulates Akt/mammalian target of rapamycin (mTOR) and its downstream targets in $\mathrm{C} 2 \mathrm{C} 12$ cells. Western blot analysis of the Akt/mTOR signaling pathway in $\mathrm{C} 2 \mathrm{C} 12$ cells. Cells were cultured with differentiation medium in the presence of LE for $24 \mathrm{~h}$, harvested, lysed and processed for western blot analysis. Band density of phosphorylated proteins was quantified by densitometry and normalized to the respective unphosphorylated proteins. Fold changes are expressed relative to the untreated controls. Results are representative of 3 independent experiments. LE, ethanol extract of loquat leaf; 4E-BP1, eukaryotic translation initiation factor $4 \mathrm{E}$ binding protein 1.

blot analysis (Fig. 5C) clearly indicated that treatment with LE increased the expression of MyoD in $\mathrm{C} 2 \mathrm{C} 12$ cells in a concentration-dependent manner. In addition, it was evident that LE induced the expression of myogenin (Fig. 5C). Overall, these results suggest that LE promotes myogenic differentiation through the upregulation of $\mathrm{MyoD}$ and myogenin.

Treatment with LE activates the Akt/mTOR signaling pathway during the myogenic differentiation of C2C12 cells. Phosphatidylinositol 3-kinase (PI3K)/Akt and its downstream mTOR pathway have been shown to play an important role in skeletal myogenesis $(26,27)$. Thus, we examined whether LE can modulate the activation of the PI3K/Akt/mTOR signaling pathway in $\mathrm{C} 2 \mathrm{C} 12$ cells. We found that LE induced the phosphorylation of Akt, p70 S6K and 4E-BP1 in a concentration-dependent manner (Fig. 6). The effects of LE on 4E-BP1 activation, however, were clearly evident and occurred in a concentration-dependent manner, whereas those on Akt and p70 S6K were not so prominent. Of note, treatment with LE induced both the phosphorylation and expression of Akt. By contrast, no significant change in the expression of mTOR was observed in the LE-treated C2C12 cells (Fig. 6). Taken together, these results indicate that $\mathrm{LE}$ enhances myogenic differentiation through the PI3K/Akt/mTOR signaling pathway; however, the effects of LE on signaling molecules vary.

\section{Discussion}

As the proportion of older subjects to younger ones is increasing worldwide, more attention is being paid toward 'healthy aging' and 'quality of life'. Although physical exercise and an adequate diet, in terms of both calorie and protein intake, contribute to the preservation of muscle function in older subjects, these strategies are limited to healthy persons rather than those who suffer from illness or inactivity (28). Therefore, in the present, we investigated whether LE has the potential to prevent the age-associated loss of muscle function. In myoblast culture and aged rats, we found that LE abrogated the decline in skeletal muscle function, including the loss of muscle mass. When examining the mechanisms involved, we found that LE enhanced myogenic differentiation through the upregulation of myogenic gene expression. We also observed that LE activated the Akt/mTOR pathway, which is a key cascade in skeletal muscle protein synthesis.

To the best of our knowledge, this is the first study to demonstrate that LE suppresses the age-associated loss of skeletal muscle mass and muscle strength in rodents. Whether LE affects the regenerative capacity of muscle fibers and the expression of myogenic proteins has not been investigated previously. However, LE is known to have therapeutic potential in diabetes and inflammation, which are closely linked to age-related muscle loss $(9,10,14,29)$. Although it is well known that certain nutritional interventions, such as essential amino acids, milk-based proteins, creatine monohydrate, essential fatty acids and vitamin $\mathrm{D}$, in combination with resistance exercise, may further enhance the beneficial effects on muscle mass and strength in aged populations (30), a small number of studies have reported the preventive effects of natural products on skeletal muscle aging. In the present study, LE ameliorated the decline in skeletal muscle function in aged rats, due to the induction of myogenesis. LE stimulated myogenic proteins, indicating a probable mechanism for the muscle regenerative potential of LE. Other natural products, such as (-)-epicatechin (Epi) and epigallocatechin-3-gallate (EGCG), have also been reported to favorably modulate muscle cell differentiation in aged animals $(31,32)$. Similarly, LE has been shown to increase the expression of myogenic genes, which is an effect that is needed for myogenic differentiation, and the regenerative effect of Epi and EGCG on aged-associated muscle function also requires the activation of myogenic progenitor cells or myogenic genes $(31,32)$. In addition, an olive oil-derived antioxidant mixture has also been reported to prevent the aging-associated loss of skeletal muscle function, although through a different mechanism (33).

We found that LE enhanced the expression of MyoD and myogenin in $\mathrm{C} 2 \mathrm{C} 12$ myoblasts. These observations are important as MyoD and myogenin are known as MRFs which play master roles in the process of generating muscle (also known as myogenesis). Myogenesis can occur during both embryonic development and post-natal life with many similarities in molecular mechanisms (34). In adult skeletal muscle, as in all renewing organs, myogenesis depends on a mechanism that compensates for the turnover of terminally differentiated cells to maintain tissue homeostasis. Therefore, myogenesis in adult skeletal muscle depends on the activation of satellite cells that have the potential to differentiate into new fibers (34). It is a multistep process that involves withdrawal from the cell cycle, the activation of muscle-specific genes and the fusion of differentiated myocytes in multinucleated myotubes. This step is controlled by the MRFs, a group of bHLH transcription factors 
composed of myogenic factor 5 (Myf5), MyoD, myogenic factor 6 (Myf6) and myogenin (35). In particular, MyoD and Myf5 function early in the commitment steps of myogenesis; myogenin and Myf6 act at later stages by promoting myoblast fusion and the differentiation of mature skeletal muscle fibers (34). Thes MRFs control the expression of structural muscle-specific genes, such as MyHC. In addition, our results demonstrated that LE is able to increase the expression level of $\mathrm{MyHC}$ and thereby potentially enhance myogenic differentiation.

Our results are also in agreement with those of previous studies indicating that the stimulation of MRFs drives myogenic differentiation. For instance, resveratrol, a polyphenol, has been shown to promote myogenic differentiation by inducing the expression of myogenin (36). Recently, the natural products betaine, kazinol-P and tetrahydropalmatine have also been shown to enhance myogenic differentiation through upregulation of MRFs (37-39).

We further demonstrated that LE activated the Akt/mTOR pathway. Akt, a seine/threonine kinase, affects several other signaling pathways that positively or negatively regulate growth, proliferation, survival and myogenic differentiation (40). A protein that is likely to have pleiotropic functions, mTOR, is best known for its role in regulating translation initiation (41). Thus, 4E-BP1 and S6K1, two of the most well characterized downstream effectors of the mTOR pathway, have been known to regulate translation initiation $(42,43)$. $4 \mathrm{E}-\mathrm{BP} 1$ and S6K1 are regulated by mTOR and the PI3K/Akt pathway in parallel (41). Notably, in this study, LE treatment did not alter mTOR phosphorylation, while an increase in the levels of phosphorylated Akt, p70 S6K and 4E-BP1 were observed with LE treatment. Although mTOR is a direct substrate for Akt, and Ser-2448 is identified as the Akt target site in mTOR, kinase-dependent and kinase-independent functions of mTOR in skeletal muscle myogenesis have been reported (26). Park and Chen (44) described that the kinase activity of mTOR was not required for nascent myotube formation, but was essential for myotube maturation. These findings may explain our results regarding mTOR; however, further mechanistic studies on the LE-induced activation of the Akt/mTOR pathway are required.

The data from the present study and our previous study on the protective effects of LE against atrophy (45) present potentially important observations with clinical implications for the population of elderly persons who suffer from impaired mobility and fragility fractures due to muscle wasting or muscle loss. Subsequent studies should be conducted to determine whether, as shown by our observations of aged rats, elderly humans will derive similar benefits from consuming LE during a period of rehabilitation following hospitalization or other inactivity. The daily consumption of up to $294 \mathrm{mg}$ of loquat leaf extract was used in a randomized double-blinded clinical study with hyperlipidemia volunteers apparently without negative side-effects (46). Assuming a $60 \mathrm{~kg}$ human, the $294 \mathrm{mg} /$ day used in that study would be equivalent to $30.2 \mathrm{mg} / \mathrm{kg}$ of $\mathrm{LE}$, which is below the $50 \mathrm{mg} / \mathrm{kg}$ used in the present study. While the analysis of ingredient composition is needed and the administration period is different, such as 35 days vs. 3 months, their result warrants further trial with LE (46). Even given the promising effects of LE, an optimal clinical trial design would need to include a dose establishment and a significant number of elderly subjects to demonstrate the desired therapeutic effects of LE, i.e., increased muscle mass, without undesirable side-effects.

\section{Acknowledgements}

This study was supported by the R\&D program of MOTIE/KIAT (N0000697; Establishment of Infrastructure for Anti-aging Industry Support) and the R\&D program of MOTIE/KEIT (10040391; Development of Functional Food Materials and Device for Prevention of Aging-associated Muscle Function Decrease). This study was also supported by the National Research Foundation of Korea (NRF) grant, funded by the Korean Government (MSIP, No. 2009-0083538). We thank the Aging Tissue Bank for providing research information.

\section{References}

1. Wernette CM, White BD and Zizza CA: Signaling proteins that influence energy intake may affect unintentional weight loss in elderly persons. J Am Diet Assoc 111: 864-873, 2011.

2. Taekema DG, Gussekloo J, Maier AB, Westendorp RG and de Craen AJ: Handgrip strength as a predictor of functional, psychological and social health. A prospective population-based study among the oldest old. Age Ageing 39: 331-337, 2010.

3. Doherty TJ: Invited review: aging and sarcopenia. J Appl Physiol 95: 1717-1727, 2003.

4. Lexell J, Taylor CC and Sjöström M: What is the cause of the ageing atrophy? Total number, size and proportion of different fiber types studied in whole vastus lateralis muscle from 15- to 83-year-old men. J Neurol Sci 84: 275-294, 1988.

5. Nilwik R, Snijders T, Leenders M, Groen BB, van Kranenburg J, Verdijk LB and van Loon LJ: The decline in skeletal muscle mass with aging is mainly attributed to a reduction in type II muscle fiber size. Exp Gerontol 48: 492-498, 2013.

6. Vinciguerra M, Musaro A and Rosenthal N: Regulation of muscle atrophy in aging and disease. Adv Exp Med Biol 694: 211-233, 2010.

7. Shefer G, Rauner G, Yablonka-Reuveni Z and Benayahu D: Reduced satellite cell numbers and myogenic capacity in aging can be alleviated by endurance exercise. PLoS One 5: e13307, 2010.

8. Uto T, Suangkaew N, Morinaga O, Kariyazono H, Oiso S and Shoyama Y: Eriobotryae folium extract suppresses LPS-induced iNOS and COX-2 expression by inhibition of NF-kappaB and MAPK activation in murine macrophages. Am J Chin Med 38: 985-994, 2010.

9. Noreen W, Wadood A, Hidayat HK and Wahid SA: Effect of Eriobotrya japonica on blood glucose levels of normal and alloxan-diabetic rabbits. Planta Med 54: 196-199, 1988.

10. Li WL, Wu JL, Ren BR, Chen J and Lu CG: Pharmacological studies on anti-hyperglycemic effect of folium eriobotryae. Am J Chin Med 35: 705-711, 2007.

11. Cha DS, Shin TY, Eun JS, Kim DK and Jeon H: Anti-metastatic properties of the leaves of Eriobotrya japonica. Arch Pharm Res 34: 425-436, 2011.

12. Alshaker HA, Qinna NA, Qadan F, Bustami M and Matalka KZ: Eriobotrya japonica hydrophilic extract modulates cytokines in normal tissues, in the tumor of Meth-A-fibrosarcoma bearing mice, and enhances their survival time. BMC Complement Altern Med 11: 9, 2011.

13. Huang Y, Li J, Cao Q, Yu SC, Lv XW, Jin Y, Zhang L, Zou YH and Ge JF: Anti-oxidative effect of triterpene acids of Eriobotrya japonica (Thunb.) Lindl. leaf in chronic bronchitis rats. Life Sci 78: 2749-2757, 2006.

14. Ge JF, Wang TY, Zhao B, Lv XW, Jin Y, Peng L, Yu SC and Li J: Anti-inflammatory effect of triterpenoic Aacids of Eriobotrya japonica (Thunb.) Lindl. Leaf on rat model of chronic bronchitis. Am J Chin Med 37: 309-321, 2009.

15. Yang Y, Huang Y, Huang C, Lv X, Liu L, Wang Y and Li J: Antifibrosis effects of triterpene acids of Eriobotrya japonica (Thunb.) Lindl. leaf in a rat model of bleomycin-induced pulmonary fibrosis. J Pharm Pharmacol 64: 1751-1760, 2012. 
16. Banno N, Akihisa T, Tokuda H, Yasukawa K, Taguchi Y, Akazawa H, Ukiya M, Kimura Y, Suzuki T and Nishino $H$ : Anti-inflammatory and antitumor-promoting effects of the triterpene acids from the leaves of Eriobotrya japonica. Biol Pharm Bull 28: 1995-1999, 2005.

17. Tan H, Furuta S, Nagata T, Ohnuki K, Akasaka T, Shirouchi B, Sato M, Kondo R and Shimizu K: Inhibitory effects of the leaves of loquat (Eriobotrya japonica) on bone mineral density loss in ovariectomized mice and osteoclast differentiation. J Agric Food Chem 62: 836-841, 2014.

18. De Tommasi N, De Simone F, Pizza C, Mahmood N, Moore PS, Conti C, Orsi N and Stein ML: Constituents of Eriobotrya japonica. A study of their antiviral properties. J Nat Prod 55: 1067-1073, 1992

19. Kunkel SD, Suneja M, Ebert SM, Bongers KS, Fox DK Malmberg SE, Alipour F, Shields RK and Adams CM: mRNA expression signatures of human skeletal muscle atrophy identify a natural compound that increases muscle mass. Cell Metab 13: 627-638, 2011.

20. Kunkel SD, Elmore CJ, Bongers KS, Ebert SM, Fox DK Dyle MC, Bullard SA and Adams CM: Ursolic acid increases skeletal muscle and brown fat and decreases diet-induced obesity, glucose intolerance and fatty liver disease. PLoS One 7: e39332. 2012.

21. Kim M, Sung B, Kang YJ, Kim DH, Lee Y, Hwang SY, Yoon JH Yoo MA, Kim CM, Chung HY and Kim ND: The combination of ursolic acid and leucine potentiates the differentiation of $\mathrm{C} 2 \mathrm{C} 12$ murine myoblasts through the mTOR signaling pathway. Int J Mol Med 35: 755-762, 2015.

22. Jung HA, Park JC, Chung HY, Kim J and Choi JS: Antioxidant flavonoids and chlorogenic acid from the leaves of Eriobotrya japonica. Arch Pharm Res 22: 213-218, 1999.

23. Nuss JE, Amaning JK, Bailey CE, DeFord JH, Dimayuga VL, Rabek JP and Papaconstantinou J: Oxidative modification and aggregation of creatine kinase from aged mouse skeletal muscle. Aging (Albany NY) 1: 557-572, 2009.

24. Novitch BG, Mulligan GJ, Jacks T and Lassar AB: Skeletal muscle cells lacking the retinoblastoma protein display defects in muscle gene expression and accumulate in S and $\mathrm{G} 2$ phases of the cell cycle. J Cell Biol 135: 441-456, 1996.

25. Dufresne MJ, MacLeod J, Rogers J and Sanwal BD: Serine auxotrophy of myoblasts in primary and secondary culture. Biochem Biophys Res Commun 70: 1085-1090, 1976.

26. Ge Y and Chen J: Mammalian target of rapamycin (mTOR) signaling network in skeletal myogenesis. J Biol Chem 287 43928-43935, 2012.

27. Ge Y, Wu AL, Warnes C, Liu J, Zhang C, Kawasome H, Terada N, Boppart MD, Schoenherr CJ and Chen J: mTOR regulates skeletal muscle regeneration in vivo through kinase-dependent and kinase-independent mechanisms. Am J Physiol Cell Physiol 297: C1434-C1444, 2009.

28. Sarti S, Ruggiero E, Coin A, Toffanello ED, Perissinotto E, Miotto F, Pintore G, Inelmen EM, Manzato E and Sergi G: Dietary intake and physical performance in healthy elderly women: a 3-year follow-up. Exp Gerontol 48: 250-254, 2013

29. Cha DS, Eun JS and Jeon H: Anti-inflammatory and antinociceptive properties of the leaves of Eriobotrya japonica. J Ethnopharmacol 134: 305-312, 2011.

30. Candow DG, Forbes SC, Little JP, Cornish SM, Pinkoski C and Chilibeck PD: Effect of nutritional interventions and resistance exercise on aging muscle mass and strength. Biogerontology 13: $345-358,2012$
31. Gutierrez-Salmean G, Ciaraldi TP, Nogueira L, Barboza J Taub PR, Hogan MC, Henry RR, Meaney E, Villarreal F, Ceballos G and Ramirez-Sanchez I: Effects of (-)-epicatechin on molecular modulators of skeletal muscle growth and differentiation. J Nutr Biochem 25: 91-94, 2014.

32. Alway SE, Bennett BT, Wilson JC, Edens NK and Pereira SL: Epigallocatechin-3-gallate improves plantaris muscle recovery after disuse in aged rats. Exp Gerontol 50: 82-94, 2014.

33. Pierno S, Tricarico D, Liantonio A, Mele A, Digennaro C, Rolland JF, Bianco G, Villanova L, Merendino A, Camerino GM, et al: An olive oil-derived antioxidant mixture ameliorates the age-related decline of skeletal muscle function. Age (Dordr) 36: 73-88, 2014.

34. Bentzinger CF, Wang YX and Rudnicki MA: Building muscle: molecular regulation of myogenesis. Cold Spring Harb Perspect Biol 4: a008342, 2012.

35. Parker MH, Seale P and Rudnicki MA: Looking back to the embryo: Defining transcriptional networks in adult myogenesis. Nat Rev Genet 4: 497-507, 2003.

36. Kaminski J, Lançon A, Aires V, Limagne E, Tili E, Michaille JJ and Latruffe N: Resveratrol initiates differentiation of mouse skeletal muscle-derived $\mathrm{C} 2 \mathrm{C} 12$ myoblasts. Biochem Pharmacol 84: 1251-1259, 2012.

37. Senesi P, Luzi L, Montesano A, Mazzocchi N and Terruzzi I: Betaine supplement enhances skeletal muscle differentiation in murine myoblasts via IGF-1 signaling activation. J Transl Med 11: 174, 2013.

38. Hwang J, Lee SJ, Yoo M, Go GY, Lee Y, Kim YK, Seo DW, Kang JS, Ryu JH and Bae GU: Kazinol-P from Broussonetia kazinoki enhances skeletal muscle differentiation via p38MAPK and MyoD. Biochem Biophys Res Commun 456: 471-475, 2015.

39. Lee SJ, Yoo M, Go GY, Hwang J, Lee HG, Kim YK, Seo DW, Baek NI, Ryu JH, Kang JS and Bae GU: Tetrahydropalmatine promotes myoblast differentiation through activation of p38MAPK and MyoD. Biochem Biophys Res Commun 455: 147-152, 2014.

40. Wilson EM and Rotwein P: Selective control of skeletal muscle differentiation by Akt1. J Biol Chem 282: 5106-5110, 2007.

41. Gingras AC, Raught B and Sonenberg N: Regulation of translation initiation by FRAP/mTOR. Genes Dev 15: 807-826, 2001

42. Gingras AC, Raught B and Sonenberg N: eIF4 initiation factors: Effectors of mRNA recruitment to ribosomes and regulators of translation. Annu Rev Biochem 68: 913-963, 1999.

43. Magnuson B, Ekim B and Fingar DC: Regulation and function of ribosomal protein S6 kinase (S6K) within mTOR signalling networks. Biochem J 441: 1-21, 2012.

44. Park IH and Chen J: Mammalian target of rapamycin (mTOR) signaling is required for a late-stage fusion process during skeletal myotube maturation. J Biol Chem 280: 32009-32017, 2005.

45. Noh KK, Chung KW, Sung B, Kim MJ, Park CH, Yoon C, Choi JS, Kim MK, Kim CM, Kim ND and Chung HY: Loquat (Eriobotrya japonica) extract prevents dexamethasone-induced muscle atrophy by inhibiting the muscle degradation pathway in Sprague-Dawley rats. Mol Med Rep 12: 3607-3614, 2015.

46. Said O, Saad B, Fulder S, Amin R, Kassis E and Khalil K: Hypolipidemic activity of extracts from Eriobotrya japonica and Olea europaea, traditionally used in the Greco-Arab medicine in maintaining healthy fat levels in the blood. Open Complement Med J 1: 84-91, 2009. 